Acta Poetica 28 (1-2)

PRIMAVERA-OTOÑO

2007

\title{
El falsete del tiempo: Marina Tsvetáieva
}

\author{
Tatiana Bubnova
}

Presentación de la poeta rusa Marina Tsvetáieva (1892-1941) con los comentarios de los poetas como Joseph Brodsky y de filólogos como S. Averintsev, y con las traducciones originales de Tatiana Bubnova. Tsvetáieva es una de las voces poéticas más poderosas del siglo xx (según Brodsky, incluso en cualquier lengua) que expresa su tiempo mediante el ritmo de la vivencia personal plasmada en un lenguaje renovador y creativo.

It's a presentation of the Russian poet Marina Tsvetaeva (1892-1941), with the comments of poets as Joseph Brodsky and philologists as S. Avérintsev, and with original translations by Tatiana Bubnova. Tsvetaeva is one of the most powerful poetic voices of the $20^{\text {th }}$ century (according to Brodsky, in any language), expressing her own time by the rhythm of the personal experience, and a creative and innovative language. 

Acta Poetica $28(1-2)$

PRIMAVERA-OTOÑO

2007

Tatiana Bubnova

IIFL, UNAM

\section{El falsete del tiempo: Marina Tsvetáieva}

Si el tiempo es una fuente del ritmo, cada poema es un tiempo reorganizado. Y cuanto más los poemas son diversificados desde el punto de vista técnico, tanto más íntimo es su contacto con el tiempo como fuente del ritmo. El tiempo habla con los individuos mediante diferentes voces. El tiempo tiene su bajo, su tenor. Y tiene su falsete. Tsvetáieva es uno de los poetas más heterogéneos desde este punto de vista. Es una poeta rítmicamente rica, generosa. Tsvetáieva es el falsete del tiempo. Una voz que sobrepasa el alfabeto musical. He aquí la traducción, débil reflejo de los ritmos férreos de lo que se encuentra transcrito en la nota al pie de página:

En vano penetro con el ojo, cual si fuera un clavo, la tierra negra.

En la conciencia, más fuerte que un clavo: aquí no estás. Y no estás.

En vano a todo el alcance del ojo escudriño la bóveda celeste.

Sólo la lluvia: un barril de agua de lluvia.

Allí no estás. Y no estás. 
No, no: ninguna de las dos:

el hueso es demasiado hueso,

el espíritu, demasiado espíritu.

¿Dónde estás? ¿Dónde está aquél? ¿Dónde está el mismo?

¿El completo?

Allá, es demasiado allá. Aquí, demasiado aquí.

No eres tú, no eres tú.

Canten lo que canten los curas,

que la muerte es vida y que la vida es muerte,

Dios es demasiado Dios, el gusano, demasiado gusano.

No es posible dividirte

en cadáver y fantasma.

No te vamos a canjear por el humo de los incensarios,

por las flores de las tumbas.

Y si en alguna parte estás,

es en nosotros. El mejor honor para vosotros,

los que os fuisteis, es despreciar el cisma.

Se fue del todo. Se fue con todo ${ }^{1}$

Recuerdo al lector que, de acuerdo con el Diccionario de la Real Academia Española, falsete quiere decir "voz más aguda que la natural, que se produce haciendo vibrar las cuerdas superiores de la laringe".

${ }^{1}$ Напрасно глазом -как гвоздем / Пронизываю чернозем: / В сознании -верней гвоздя: / Здесь нет тебя -и нет тебя. // Напрасно в ока оборот / Обшариваю небосвод: / --Дождь! Дождевой воды бадья. / Там нет тебя -и нет тебя. // Нет, никоторое из двух: / Кость -слишком кость, дух слишком дух. / Где --ты? Где -тот? Где -сам? Где -весь? / Там -слишком там, здесь -слишком здесь. // Не подменю тебя песком / И паром. Взявшего -родством / За труп и призрак не отдам. / Здесь -слишком здесь, там -слишком там. // Не ты -не ты -не ты -не ты. / Что бы ни пели нам попы, / Что смерть есть жизнь и жизнь есть смерть,-- / Бог слишком бог, червь -слишком червь. // На труп и призрак -неделим! / Не отдадим тебя за дым / Кадил, / Цветы / Могил. // И если где нибудь ты есть - / Так -в нас. И лучшая вам честь, / Ушедшие -презреть раскол: / Совсем ушел. Со всем -ушел. 
Para Joseph Brodsky —extraordinario poeta ruso, premio Nobel de literatura 1987-, Marina Tsvetáieva es el poeta más importante del siglo xx. No entre las mujeres poetas. No entre los poetas rusos del siglo $\mathrm{Xx}$, sino de todo el siglo Xx poético. No es mi intención imponer la opinión de Brodsky. No se trata de dar calificativos, de evaluar -quién fue mejor o más grande-, sino de darle un rango de importancia a esta poeta tan poco conocida en México, apoyándome en la opinión de Brodsky. Marina Tsvetáieva está traducida a las principales lenguas europeas. ${ }^{2}$

Ahora volvamos a la voz, al timbre poético que, en combinación con el ritmo, mencionado por Brodsky, es tal vez la característica más relevante de su poesía.

Marina a menudo empieza un poema con un $d o$ sobreagudo... Tal fue la calidad de su voz que su discurso empieza siempre desde el otro extremo de la octava, en el registro superior, en un límite donde sólo es pensable un descenso o, en el mejor de los casos, seguir en el mismo nivel. Pero el timbre de su voz era tan trágico que garantizaba la sensación de ascenso sin tomar en cuenta la duración del sonido. Lo trágico no le llegó después, en su biografía: había existido desde antes. Su biografía sólo coincidió con lo trágico y le respondió como un eco.

He aquí un poema de 1916 en el que este ascenso exclamativo e irrefrenable es patente, así como esta mirada poética desde la altura, desde la elevación por encima de los asuntos terrenales, altura desde la cual visualiza los "inflamados pleitos humanos": 3

¡Yo sé la verdad! ¡Fuera las verdades antiguas!

¡Gente contra gente no pelee más en la tierra!

\footnotetext{
${ }^{2}$ Cf. Tatiana Bubnova, Contrapunto a cuatro voces en los caminos de aire, México, UNAM, 1992, antología que contiene una sección dedicada a Tsvetáieva.

${ }^{3}$ Cita de un poema de Osip Mandelstam.
} 
Mirad: ¡ya es tarde! Mirad: ¡ya viene la noche!

¿Qué estáis disputando, poetas, amantes, guerreros?

Se extiende el viento, el suelo se cubre de rocío.

Pronto la nevasca estelar congelará al cielo.

Pronto dormiremos todos bajo la tierra,

los que no dejamos dormir al prójimo en la tierra.

La clave para entender los rasgos más importantes de la obra de Tsvetáieva es, pues, la percepción trágica de la realidad, plasmada en la exclamación poética, percepción que fue relacionada, aunque no demasiado estrechamente, con los elementos concretos de la época y la geografía. La adopción de la "nota sobreaguda" no viene a ser un capricho o una falta de gusto, sino que está producida por la altura y la implacabilidad de las exigencias éticas de la poeta hacia el tiempo y hacia sí misma. Tsvetáieva tal vez sea el poeta más sincero del siglo $\mathrm{xx}$, pero esta sinceridad, en palabras de Brodsky, es ante todo la sinceridad y la calidad genuina de la dicción poética y del sonido: "como cuando se grita de dolor. El dolor es biográfico, pero el grito es extrapersonal".

Aquí es pertinente evocar la manera como Marina Tsvetáieva concebía la poesía. Para ella, el torrente versal es semejante a la naturaleza, con la excepción de que en la creación está presente el elemento ético, de modo que el poeta es responsable por su discurso, por lo que escribe. La escritura-grito se vuelve el acto más sufrido y comprometido de la vida. Sin duda, ella suscribiría la opinión del mismo Brodsky:

La poesía no es diversión, ni siquiera una forma de arte. Es más bien nuestra finalidad en cuanto especie. Si aquello que nos distingue del resto del reino animal es el habla, entonces la poesía es la forma superior de habla, nuestra, digamos, diferencia genética principal de los animales. Al rechazarla, nos condenamos a las formas inferiores de la comunicación, tráte- 
se de la política, el comercio etc. La poesía es un colosal acelerador de la conciencia, tanto para el que la escribe como para el que la lee. En ella se ponen de manifiesto los vínculos y las dependencias en la lengua y el habla cuya existencia antes era imperceptible. Es un instrumento de cognición único.

Para el mismo Brodsky, la capacidad de ver un sentido donde por lo visto el sentido no existe es un rasgo profesional del poeta. En esta lógica, escribe Tsvetáieva, los versos no necesitan una clave para la comprensión, sino que ellos mismos son una clave para leer todo. "Pero de la comprensión a la aceptación no es que exista sólo un paso, sino que no hay ningún paso: comprender es aceptar, comprender es aprehender, ninguna otra comprensión existe, cualquier otra comprensión es incomprensión". Ser poeta: dar sentido al mundo. Otra forma de expresarlo es la de Osip Mandelstam, contemporáneo y amigo de Tsvetáieva: "La poesía es la conciencia de tener razón".

Ser poeta, desde este punto de vista, es una forma de vivir y de construir la vida propia. Coincide con la idea del romanticismo de convertir la vida propia en una obra de arte o, mejor, en parte del proceso creativo. Así, para Osip Mandelstam, "la muerte de un artista no es una casualidad, sino un último acto de creación que ilumina, como un haz luminoso, todo el camino de su vida". La identificación del arte con la vida, la fusión inmediata del arte con la vida, la construcción de la vida propia como una obra de arte son marcas distintivas del romanticismo (acuérdense de la poesía y la vida de Byron, y sobre todo de su percepción como paradigma romántico en el siglo XIX). En esta perspectiva se puede decir que Tsvetáieva fue una poeta romántica. El viejo principio romántico, de acuerdo con el cual un poeta está obligado a no salir del éxtasis las 24 horas del día, incinerándose y quemándose a la vista de todos como una vela inextinguible, no era pose, sino el motivo recurrente en la poesía rusa de los principios del siglo xx, época 
final del simbolismo. Fue la época del extraordinario florecimiento cultural, en particular literario, llamado ahora "siglo de plata" (por contraste con el "siglo de oro" de la poesía rusa, que cae en el primer tercio del XIX). Por cierto, durante el siglo de plata poético estas actitudes resultaban bastante comunes. "Extiende tus brazos y enciéndete", "Te amo porque estás encendido", escribe Viacheslav Ivanov. Maiakovski habla del "incendio del corazón". Andrei Biely exclama: "Solloza, elemento tormentoso, / en las columnas de los fuegos y truenos. / ¡Rusia, Rusia, Rusia, / incinérame, demencial!” Metáfora que Rusia no dejó de materializar muy pronto con los mejores representantes de su cultura. "La imagen trágica de Blok, la santa locura mortal de Jlébnikov, los modales de torero suicida de Maiakovski, son ejemplos de esta combustión permanente, que no es una pose, sino una utopía", dice S. Averintsev. La última víctima en este altar fue, desde luego, Tsvetáieva, que pudo canalizar su sistema poético hacia recursos más contemporáneos, pero dejó intacta la orientación individualista de los simbolistas. El simbolismo fue, como lo muestra José Ricardo Chaves, una continuidad y permanencia de la actitud romántica hacia la vida y la creación. Esta actitud encuentra su eco en las líneas de Marina Tsvetáieva, contemporánea de los últimos simbolistas, líneas que subrayan su capacidad de convertir en materia poética todo lo que sea inservible para tales propósitos en la opinión de otros; el elemento del fuego está presente, siendo la misma autora el fuego de la acción creadora:

¡Traedme todo aquello que a otros no hace falta!

Todo se ha de abrasar en mi fuego.

Atraigo a la vida y a la muerte atraigo, ambas son dones para mi fuego.

La llama se aviva con objetos livianos:

ramas secas del año pasado, coronas de flores, palabras. 
¡La llama estalla con semejante alimento!

¡Os levantaréis más puros que la ceniza!

¡Soy el ave Fénix, sólo en el fuego canto!

¡Sostened, conservad mi elevada suerte!

Me abraso en la altura, me quemo hasta ser pavesas,

¡que la noche os sea más luminosa!

¡Hoguera de hielo, fuente de fuego!

En alto sostengo mi erguido porte,

en alto mantengo mi sublime jerarquía:

¡soy la Interlocutora, soy la Heredera!

La vida real le ofreció a Tsvetáieva innumerables oportunidades para vivirla en esta clave: su existencia fue marcada muchas veces por la tragedia, hasta que una de estas vueltas patéticas y trágicas de la vida la lleva al suicidio. Nacida en 1892 en una familia relacionada con el arte - la madre era una talentosa pianista; el padre, filólogo e historiador de arte, fue fundador y curador de los dos museos de arte más importantes de Moscú-, Marina, en contra de las aspiraciones de la madre, que pretendía llevarla por la vía de la música, desde muy temprano manifestó una vocación poética, de modo que a los 18 años ya fue posible la publicación de un primer libro que fue recibido en el ambiente literario moscovita de una manera generosa. En este contexto hay que mencionar que justamente en esta época — estamos hablando de 1910—, en el horizonte poético ruso se eleva una estrella de primera magnitud que es Anna Ajmátova, cuyo reconocimiento fue asimismo rápido y unánime. En el período inicial de su carrera creativa, Marina reconocía plenamente la prioridad de Ajmátova (1889-1966). Desde muy joven vivía en la disciplina creativa de escribir permanentemente, convirtiendo las experiencias cotidianas en líricas: una enorme porción de su poesía, sobre todo de la temprana, tiene este carácter de diario lírico, y el volumen de 
su producción poética es considerable, sobre todo comparándolo con el de sus contemporáneos equipolentes Mandelstam (1891-1938), Pasternak (1890-1960), Ajmátova (a los que es necesario poner en la misma serie con Tsvetáieva, tanto por motivos biográficos como por la relevancia y calidad poética). Hay que recordar que la existencia de esta generación de creadores fue marcada por las grandes tragedias históricas. Les tocó vivir tres revoluciones (una en 1905-1907, otras dos en 1917), la Guerra civil, la Primera y la Segunda guerras mundiales, el terror estalinista. Marina Tsvetáieva vivió en el abandono, con sus dos hijas, el período posrevolucionario de la guerra civil y la destrucción del país (1918-1922), padeciendo la hambruna y toda clase de privaciones, en la Moscú de los Soviets, mientras su marido participaba en la guerra civil del lado "equivocado", es decir, en el ejército blanco, contrarrevolucionario. Su hija menor muere de hambre en 1920, suceso que Tsvetáieva no pudo perdonarse a sí misma durante toda su vida. Emigra con su hija mayor, para encontrarse con su marido sobreviviente, en 1922, primero a Berlín, luego a Checoslovaquia y, finalmente, a Francia. Escribe permanentemente, y publica cierta porción de su obra en la prensa de los emigrados rusos, con los cuales, sin embargo, entra en un conflicto y es aislada debido a su actitud, en la opinión de ellos ambigua, hacia la Unión Soviética. Esta situación se acentuó aún más con los acontecimientos posteriores. En los años treinta su marido, ex guardia blanco, se acerca a las organizaciones de emigrados que buscan un acercamiento a la Unión Soviética y termina por colaborar con la Seguridad soviética (NKVD: Comisariato Popular de Asuntos Internos, más tarde conocido como KGB: Comité de Seguridad del Estado), en la organización de los asesinatos políticos (estuvo, en particular, involucrado en el asesinato del hijo de Trotsky). Los hijos de la pareja y el marido (Sergei Efrón) están persuadidos de que la familia debe regresar a Rusia, a pesar de que Tsvetáieva no está nada con- 
vencida de la urgencia de dar este paso. No obstante, la necesidad externa acelera el regreso (Sergei Efrón, involucrado en otro asesinato político, es perseguido por la policía francesa), y primero la hija mayor de Tsvetáieva, Ariadna Efrón, luego Sergei Efrón se van a la Unión Soviética. Es el peor momento para el regreso (se acerca el terrible año de las represiones y purgas estalinistas masivas, el fatídico 1937), pero el marido y los hijos han idealizado las transformaciones en el país y no tienen ni la menor idea de lo que enfrentarían en el pico de las persecuciones estalinistas. En 1938, Marina con el hijo menor Georgi (Jorge), nacido en Checoslovaquia en 1925, regresan también a la patria, para presenciar, en pocos meses, el arresto de Ariadna y, poco después, el de Sergei. Aislada en el ambiente de la cultura soviética en su calidad de familiar de los supuestos contrarrevolucionarios, sin recursos para subsistir, Tsvetáieva lleva una vida aún más miserable que en la emigración, donde la familia sufrió muchas penurias y privaciones. En 1941 empieza la guerra de Alemania contra la Unión Soviética. Forzada a evacuar hacia las regiones remotas, pobres e incultas, acosada por el ambiente que la rodea y por los órganos de seguridad, sin medios para vivir y en conflicto permanente con el hijo menor, en el contexto del avance que parecía irrefrenable de las tropas alemanas hacia el corazón de Rusia, Marina Tsvetáieva se suicida el 31 de agosto de 1941.

Acerca de su relación con la patria conviene oírla a ella misma: "La Patria no es un territorio convencional, sino la irrevocabilidad de la memoria y de la sangre. No estar en Rusia, olvidar a Rusia: temerlo sólo puede aquél que piensa a Rusia como algo externo a su persona. Quien la lleva adentro, la perderá sólo con su vida".

Durante su estancia en Francia escribe el siguiente poema: 
¡Nostalgia de la patria!

Superchería hace tiempo

denunciada. A mí

me es absolutamente

igual

dónde estar

tan absolutamente sola,

sobre cuál

empedrado caminar

cargando la canasta de mercado,

hacia una casa que

(¿cuartel u hospital?),

si es mía,

ella no lo sabe.

Me da igual

entre qué caras he de mostrar

mi gesto de león cautivo,

qué medio humano

me va a desplazar — $i$ sin falta! -

al fuero íntimo,

a la privacía del sentido.

A mí, oso polar sin témpano, me da

lo mismo dónde nivelarme (¡qué va!),

o dónde humillarme.

Tampoco me seduce el llamado

materno de mi lengua.

$¡$ Me es indiferente

en cuál

me desentienda con quien sea!

(Con el lector

devorador de toneladas de papel

periódico, ordeñador de chismes...

A este siglo pertenece él, pero yo

soy anterior a todo siglo).

Como madero, encallada quedé 
donde había una arboleda.

Me da lo mismo todo,

todo me es igual,

y más indiferente $-\mathrm{y}$ querido-

lo que ya se fue.

Perdí las señas de identidad,

las fechas y los rasgos. Alma soy

nacida alguna vez en algún lugar.

Y tanto mi país me descuidó,

que ni el fisgón más avispado

me podrá hallar

—en el haz y en el envés del alma-

marcas del nacimiento.

Todas las casas son ajenas para mí, vacíos para mí son todos los templos.

Me da lo mismo todo, todo me es igual.

Pero si encuentro por el camino un arbusto,

y sobre todo si se trata de un serbal... $(1934)^{4}$

Ahora bien, en opinión de muchos, los acontecimientos externos son sólo un pretexto para vivir la creación en una clave trágica. Marina buscaba en todo la plenitud de vivencias, vivía una permanente orgía de sentimientos que plasmaba inmediatamente en versos. "Tsvetáieva era una mujer de activa alma masculina, decidida, guerrera, indómita. Lo mismo en su vida que en su obra, y de manera impetuosa, ávida y casi voraz, buscó lo definitivo y lo terminante, y en perseguirlo llegó muy lejos, adelantándose a todos", escribió Boris Pasternak (18911960), poeta que fue vinculado con ella por una correspondencia poética en la que se mezclaba el arranque lírico con el erotismo que se convertía igualmente en poesía, su cómplice intelectual y amigo inconstante, que, como otros objetos amorosos de Marina, la decepcionó. Eso del alma masculina men-

\footnotetext{
${ }^{4}$ Por cierto, el serbal (que no es un arbusto, sino un árbol), motivo folklórico de las canciones populares, es para Tsvetáieva el símbolo de la patria.
} 
cionada por Pasternak no debe confundirnos, la de Tsvetáieva, como la de Ajmátova, es la poesía de mujer poeta capaz de elevar la experiencia específicamente femenina, a través del crisol de la creación, a una experiencia de alcance universal. No obstante, la esencia de la poesía no tiene sexo o es, más bien, andrógina, como lo es la poesía de Tsvetáieva y como es, en sus mejores momentos, la de Ajmátova.

"La voz de Tsvetáieva es la de la tragedia. Expresa un malestar colosal. ¿Es Job hombre o mujer? —se interroga Brodsky-. Tsvetáieva es un Job con faldas". Lo femenino tal vez se manifiesta en su poesía en una mayor sensibilidad hacia los aspectos éticos. Y en primer lugar, Tsvetáieva es en este aspecto muy exigente hacia sí misma. Su vida tiene que ser una combustión en la flama amorosa, y su exigencia hacia los objetos de su pasión es tan intensa, idealizante y demandante que estos objetos por lo general son incapaces de tolerar esta intensidad. Así, uno de sus amantes concebía el amor, según la misma Tsvetáieva, en un plano horizontal, terrenal, remitiéndolo al mundo de las preocupaciones y necesidades cotidianas, mientras que para ella el amor tenía que ser vertical, metafísico, como algo que interpela la eternidad. Entre sus amados, que no han podido tolerar su fuego, hay que contar con el ya mencionado Pasternak y Rainer Maria Rilke, con quien se comunicaba por medio de cartas. Rilke le dedicó una elegía: “¡Las pérdidas en el Mundo, Marina, las estrellas que caen!"5 Lo relevante de estas situaciones recurrentes de apasionamiento y decepción es que la experiencia se transforma en una poesía de la más alta ley.

Te arrebataré a todas las tierras o cielos, porque el bosque es mi cuna, y el bosque es mi sepulcro,

\footnotetext{
${ }^{5} \mathrm{La}$ correspondencia entre los tres, traducida al español, fue publicada por Siglo XXI en 1986 (Cartas del verano de 1926).
} 
porque con un solo pie apenas piso la tierra, porque sé cantar para ti como nadie sabe.

Te arrebataré a todos los tiempos y noches, a todas las doradas banderas, a todas las espadas, esconderé las llaves, echaré de la entrada a los perros, porque en la noche invernal soy más fiel que un perro.

Te arrebataré a todas las demás y a aquélla, la única, no serás prometido de nadie, no seré la esposa de nadie. Y en la última contienda te quitaré — iguarda silencio!a aquél con quien Jacob luchó en la noche.

Pero hasta que te quedes con los brazos en cruz sobre el pecho, —imaldición! — tú te has de quedar contigo.

Tus dos alas, dirigidas hacia el éter, iporque el mundo es tu cuna, y el mundo es tu sepulcro!

¿Cómo era Marina Tsvetáieva? He aquí la descripción de su hija Ariadna Efrón:

Tenía un porte severo y esbelto. Incluso inclinada sobre el escritorio conservaba la tensión del acero en el espinazo. Su cabello, de un castaño dorado, en la juventud era ondulado, pero empezó a encanecer temprano, lo cual fortalecía la sensación de la luz que irradiaba su rostro moreno y pálido, mate; los ojos eran claros y permanentemente encendidos, verdes, de color de uva, rodeados de unos párpados cafés. Su cara, que parecía acabada hasta una completud, hasta una estaticidad, estaba plena de un permanente movimiento interno, de expresividad oculta, cambiante y saturada de matices, como el cielo y el agua. Pero pocos eran los que sabían leer esta cara.

Era activamente generosa y bondadosa; se apresuraba a ayudar, a sacar de problemas, a salvar, o tan sólo a poner el hombro. Compartía lo último y lo más esencial que tenía, puesto que no poseía nada superfluo. Sabiendo dar, sabía también tomar sin ceremonias; durante mucho tiempo creía en la 
efectividad de la 'caución solidaria del bien', 6 en la gran ayuda mutua inextinguible de los seres humanos. Nunca estuvo desamparada, pero siempre indefensa. Condescendiente con los ajenos, de los prójimos —amigos, hijos— exigía, como de sí misma, de una manera exagerada.

Odiaba la cotidianidad — por su carácter inacabable, por la repetición inútil de las preocupaciones diarias, por devorar el tiempo necesario para lo principal- La superaba con paciencia y distancia durante toda su vida.

Era una persona de la palabra, de la acción, del deber. A pesar de toda su modestia conocía su propio valor.

Tsvetáieva escribía acerca de sí misma: "Mi desgracia consiste en que para mí no existe ninguna cosa externa, sino que todo es corazón y destino". Se caracteriza a sí misma: "Un alma que no conoce la medida".

Entre otras opiniones contemporáneas, destaca la de la viuda del poeta N. Mandelstam: "Marina en todo buscaba éxtasis y plenitud de sentimientos. Necesitaba el éxtasis no sólo del amor, sino también del abandono, olvido, desgracia... En una orientación así veo una extraña nobleza, pero me inquieta la indiferencia a la gente relacionada con ella, gente que en un momento dado no necesita o impide la orgía de sentimientos". En efecto, tanto en la vida propia como en la aceptación de la de los demás, Tsvetáieva tendía a prescindir de los aspectos relacionados con la existencia cotidiana, y era capaz de pasar por alto los problemas y sentimientos de otros, si eran contrarios a su propio estado de ánimo. Como identificaba, según he dicho, el vivir con la poesía, sus demandas a la vida común, a la que pretendía imponer un nivel muy elevado de vivencias y pensamientos, eran desmesuradas, y la gente común con dificultad estaba dispuesta a tolerar su intensidad. Incluso la gente no tan ordinaria, como Pasternak, al toparse con ella en la vida

\footnotetext{
${ }^{6}$ Referencia al tema tratado por Tsvetáieva en uno de sus ensayos.
} 
real, retrocedía. Cabe hablar del gigantismo emocional de la poeta, que era una forma de vida y no un estado transitorio o fingido.

Cito un díptico de poesías que ilustra la manera en que ella era capaz de concebir el amor: en el primer poema Tsvetáieva habla de parte de Magdalena, o de una personificación de Magdalena, en el segundo, le responde Jesucristo. Por cierto, este tema de la voz de Magdalena lo retomaría veinte años después, ya muerta Tsvetáieva, Borís Pasternak en otro poema, que no viene al caso citar aquí; lo menciono para subrayar la continuidad de los temas y vivencias que se puede encontrar en la poesía de ambos. Por otra parte, entre los poemas de Marina hay algunos dedicados a Ajmátova con una exaltación y una devoción muy grandes, y unos escritos a Mandelstam, con quien también la unieron vínculos emotivos.

MAGDALENA

I

Entre nosotros, los diez mandamientos, el calor de las diez hogueras.

La sangre hermana causa rechazo, pero eres de sangre ajena.

En los tiempos evangélicos yo sería una de aquéllas... (¡La sangre ajena es la más deseada, y entre todas, la más ajena!)

Con todas mis desazones, preclaro, arrastrándome, te seguiría. Oculta la mirada demoníaca, perfumes en ti vertería:

sobre tus pies, bajo tus pies, o derramándolos a tu paso... 
¡Fluye, pasión envilecida, empeñada a los parroquianos!

Fluye con la espuma de la boca, con el fervor de la mirada.

Fluye en el sudor del lecho. Tus pies en mi cabellera calzo como en una piel.

A tus pies, como seda, me extiendo. ¿No serás aquél (¡soy aquélla!) que dijo a la bestia de la melena ígnea: “Levántate, hermana!?”

Por tus derroteros no pregunto, porque, amada, todo se cumplió. Tú me has calzado a mí, descalzo, en el torrente de tu cabello y de tu dolor.

No pregunto cuánto han costado estos perfumes. Al desnudo, a mí, con la ola de tu cuerpo me has vestido, como con un muro o una vid.

Dócil y dulce, como nunca antes, manso tocaré tu desnudez. A mí, tan recto, me has enseñado el declive de la ternura al caer a mis pies. 
Me harás una fosa entre tu pelo, y sin lienzos me envolverás.

¿Para qué me has de traer la mirra?

Como ola,

tú me lavarás.

Para Tsvetáieva el amor en la poesía - y la poesía es la vida- era inherente a la existencia misma. A pesar de la abundancia de las imágenes carnales, como en estos poemas, sus amores mayormente, por principio, eran platónicos (aunque no todos). Para ella, el encuentro real con el objeto de su pasión podía ser indeseable, por lo inevitablemente decepcionante. "Conozco un solo género de amor feliz, escribía Tsvetáieva en 1931. El de Bettina hacia Goethe. El de la gran Teresa hacia Dios. Un amor sin respuesta. Sin esperanza. Sin el estorbo de una mano que acepta. Un amor que cae como en un barril sin fondo". Y en otro lugar: "Es difícil que se me encuentre una pareja, y no es porque escribo versos, sino porque fui pensada sin pareja, el estado emparejado es para mí contra natura: alguien sobra, las más veces sobro yo..." Y es que, dice Tsvetáieva, el amor recíproco no es apropiado para ella, "el amor recíproco siempre lo sentí como un callejón sin salida: como si dos se toparan uno con otro, y todo se detiene". "El ser humano está planeado para estar solo. Donde hay dos, hay mentira”. ¿A qué responde más fervientemente la Musa de Tsvetáieva? En este caso, se imponen dos hipóstasis, dos fases del amor: su nacimiento y el dolor de la separación. En cuanto a la realización, sólo contamos con una sórdida línea: "en medio del amor hay un vacío". Hay una decidida preferencia de la plenitud de los deseos a la vacuidad de la felicidad.

Ella rechazaba activamente la vida cotidiana, en la que por cierto fue irremisiblemente sumergida como madre de familia de muy escasos recursos, pero cumplida y responsable. La cotidianidad le repugnaba y sobre todo la presionaba, quitándole 
el tiempo precioso para lo esencial: su poesía. Este eterno conflicto entre el rol socialmente aceptado de mujer en la vida cotidiana y el quehacer poético está expresado en varios lugares de su obra creativa:

Calla, encomio, no azotes la puerta.

Gloria: esquina

del escritorio y el codo.

¡Para, ajetreo!

¡Corazón, sosiégate!

El codo y la frente.

El codo y el pensamiento.

La juventud, para amar,

la vejez, a calentarse.

No hay tiempo para ser,

no hay dónde quedarse.

Un rincón tan siquiera,

pero $¡$ sin gente!

Los grifos gotean, rechinan los muebles,

las bocas hablan:

de comida llenas

te agradecen

la "belleza".

Si supierais vosotros,

el que está cerca, el de lejos, cómo compadezco

mi propia cabeza-

¡Dios en la horda!

La estepa: ¡cárcel!

El paraíso es dónde

¡nadie habla!

El mujeriego: bestia,

el tendero: ¡detalles!

Dios será para mí

aquel que me regale 
(¡no demores! los días

contados están)

cuatro paredes

llenas de paz.

O en otro lugar:

$\mathrm{Al}$ diablo el piso: tanta basura.

¿Mal la barrí? Muera la censura,

muera la escoba, muera la rutina.

El poeta pende de puntos suspensivos.

Nunca logró tener, en su vida familiar de emigrada, unas condiciones dignas para poder trabajar en su poesía: tenía que buscar oportunidades, un lugar y un tiempo libres de las tareas domésticas. Pero, con una insistencia casi maniática, Marina escribe y produce, en la emigración, lo mejor de su poesía. La innovadora y reformadora del verso, en el que logra introducir las entonaciones conversacionales y los giros populares que aprendió en las calles de Moscú durante la época revolucionaria, escribe su propia vida y versifica el proceso de la creación en unos versos vertiginosos y llenos de intensidad y pasión.

La poesía ciertamente era un escape momentáneo hacia un espacio metafísico, espiritual (o según Brodsky, hacia un vector centrífugo), que marcaba su orientación a "una verdad celeste contra la verdad terrenal". La posición vital de Tsvetáieva era una negación firme, una no aceptación de las bases de la organización de la vida, tal y como ella la percibía. El rechazo activo del orden del universo, manifiesto desde sus etapas creativas más tempranas, se reafirma debido a las circunstancias desastrosas de su vida, tanto personales como sociales en general. Entre otras decepciones, se puede mencionar, por ejemplo, el hecho de que ella tenía una devoción profunda hacia la cultura alemana desde la infancia (la alemana y la fran- 
cesa eran para ella segundas lenguas, y conocía íntimamente sus respectivas culturas), lo cual le agregó frustraciones al principio de la Segunda guerra mundial, durante la ocupación de Checoslovaquia por tropas fascistas alemanas; Checoslovaquia era para ella un país adoptivo muy entrañable. A este período se refieren las líneas que plasman este rechazo hacia el mundo, la no aceptación condensada en una situación histórica concreta, pero que de hecho iba más allá de la circunstancia, hacia una orientación metafísica general:

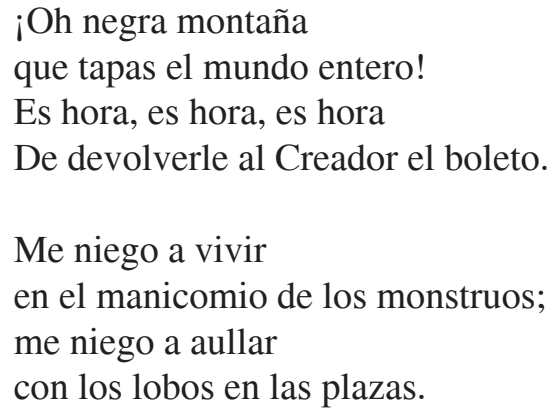

Con lo del "boleto del Creador", Tsvetáieva evoca el pasaje de Los hermanos Karamazov de Dostoievski donde Iván pretende devolverle a Dios el "boleto" para entrar en el reino de la futura felicidad universal, pero comprada al precio del sufrimiento de los inocentes. En el contexto de la invasión de las tropas fascistas a Checoslovaquia, Marina habla de devolver su propio boleto, en 1938. Pero todavía faltaba para llegar a los años siguientes, los del regreso a la patria, cuando fueron arrestados su hija y su marido, y al año 1941, el inicio de la guerra para Rusia.

Intrépida innovadora del sistema poético en los aspectos formales, más profunda que cualquier otro poeta del siglo $\mathrm{xx}$, reformadora y creadora de un lenguaje poético basado en el coloquialismo, pero aplicado a las materias metafísicas, Tsvetáieva valoraba en la poesía los aspectos éticos fundiéndolos 
con los estéticos. Así, en los años veinte, en la emigración, escribe el ensayo "El arte a la luz de la conciencia".

El estado de la creación es el estado de alucinamiento. Antes de empezar es obsesión, hasta terminar es posesión. Algo, alguien te invade, tu mano es la ejecutante, no de ti, sino de aquél. ¿Quién es él? Aquello que quiere ser a través tuyo. A mí las piezas siempre me escogían por la señal de la fuerza, y las solía escribir a menudo casi contra la voluntad. Todas mis piezas rusas son así. Ciertas cosas de Rusia querían ser dichas, y me escogieron a mí. Y me convencieron, me sedujeron - ¿con qué? - con mi propia fuerza: ¡sólo tú! [...] El estado de creación es un estado de sueño, cuando al someterte a una necesidad desconocida, incendias la casa o empujas de la montaña a un amigo. ¿Es tuyo este acto? Evidentemente es tuyo (duermes y sueñas tú). Tu acto en plena libertad, acto tuyo sin conciencia, acto tuyo de ti-naturaleza.... Pero ¿en qué se diferencia una obra de arte de una obra de la naturaleza, un poema de un árbol? [...] La tierra, que da nacimiento, es irresponsable, mientras que el hombre creador es responsable. [...] Es responsable por la creación de sus manos.

El espacio de su poesía era, para su tiempo, ubicado en la altura demasiado grande, con un aire demasiado enrarecido, exigía esfuerzo demasiado desmedido para poder existir en él. Este aspecto de elevación del mundo terrenal, este dispararse hacia un espacio metafísico, explican la devoción que Brodsky, un poeta metafísico por excelencia, sentía por ella. En este sentido, Tsvetáieva se le adelantaba al lector medio en varias generaciones, y es por eso que resulta justamente ahora tan contemporánea.

La escritura de Tsvetáieva es una escritura libre, moderna, aunque encerrada en un cinturón estrecho de la rima y sobre todo del ritmo, interpretados, por lo demás, de una manera sumamente innovadora y liberada. Con toda la orientación de su 
poesía hacia una percepción por medio del ojo, en la lectura, la misma estructura y desarrollo del verso se dictan en muchos aspectos no sólo y no tanto por la lógica del sentido, sino por la lógica del sonido, lógica de la consonancia. Aportó muchísimo a la poética rusa del siglo xx, con sus ritmos "invencibles" (en palabras de Andrei Biely), con su utilización novedosa de las asonancias, poco usadas en el verso ruso tradicional, con su uso muy amplio del encabalgamiento - de la ruptura espontánea de la sintaxis, de la frase, para transportarla a la línea siguiente o incluso a la estrofa siguiente - , de modo que la división entonacional y frástica del verso entra en contradicción con la métrica, aportando un efecto complementario de dificultad, tensión y, al mismo tiempo, de espontaneidad, de improvisación discursiva. La variedad de los ritmos que ella aportó a la poesía en lengua rusa es realmente extraordinaria.

Para Tsvetáieva eran importantes la colaboración del escritor y del lector y su entendimiento mutuo, la importancia de una colaboración: en esto también se adelantó muchísimo a su tiempo. Escribe: "Qué es la lectura, si no la adivinación, interpretación, manifestación de lo secreto, de lo que quedó más allá de las líneas, de los límites de las palabras... Si te cansas de mi pieza, quiere decir que leíste bien y que leíste algo bueno. El cansancio del lector no es un cansancio devastador, sino creador, participativo, y hace honor tanto al lector como a mí”. Termino con la traducción ${ }^{7}$ de otro poema de Tsvetáieva, en el que ella una vez más identifica la creación con la vida:

Me abrí las venas: sin parar, irreprimible, brota la vida. Poned recipientes: no encontraréis un vaso lo bastante hondo.

${ }^{7}$ Todas las traducciones de este ensayo son mías. 
Rebasará el borde,

fluirá hacia fuera:

a la negra tierra,

a nutrir la hierba.

Irreversible, incontenible,

irrestañable

el verso brota. 
